Editorial

\title{
Micro/Nanofluidic Devices for Single Cell Analysis
}

\author{
Tuhin Subhra Santra ${ }^{1}$ and Fan Gang Tseng ${ }^{1,2,3, *}$
}

1 Institute of Nano Engineering and Microsystems, National Tsing Hua University, Kuang Fu Road, No. 101, Section 2, Hsinchu 30013, Taiwan; E-Mail: santra.tuhin@ gmail.com

2 Department of Engineering and System Science, National Tsing Hua University, Kuang Fu Road, No. 101, Section 2, Hsinchu 30013, Taiwan

3 Division of Mechanics, Research Center for Applied Sciences, Academia Sinica, Nankang, 11574, Taipei, Taiwan

* Author to whom correspondence should be addressed; E-Mail: fangang@ess.nthu.edu.tw; Tel.: +886-3-571-5131 (ext. 34270); Fax: +886-3-573-3054.

Received: 28 March 2014 / Accepted: 28 March 2014 / Published: 3 April 2014

The Special Issue of Micromachines entitled "Micro/Nanofluidic Devices for Single Cell Analysis" covers recent advancements regarding the analysis of single cells by different microfluidic approaches. To understand cell to cell behavior with their organelles and their intracellular biochemical effect, single cell analysis (SCA) can provide much more detailed information from small groups of cells or even single cells, compared to conventional approaches, which only provide ensemble-average information of millions of cells together. Earlier reviews provided single cell analysis using different approaches [1-3]. The author demonstrates invasive and noninvasive with time and non-time resolved SCA [1]; whereas some other literature provided destructive (with dyes, DNA, RNA, proteins and amino acids) and nondestructive (electroporation, impedance measurement and fluorescence based methods) cellular content analysis using microfluidic devices [3]. Further literature also suggest that single cell analysis is possible with capillary electrophoresis (CE) combined with a detection method such as electrochemical detection (ED), laser induced fluorescence (LIF) detection and mass spectrometry (MS) [4,5].

This special issue mainly focuses on the recent development of SCA with different microfluidic devices based technologies. Wiklund et al. [6] reviewed the cell to cell interaction in multi-well microplates combined with live cell fluorescence microcopy by using an ultrasound method. They describe the interaction between natural killer (NK) cells and cancer cells at an individual level. This review not only elucidates on the heterogeneity in cytotoxicity of NK cells and their ability to form one or several immune synapses simultaneously, but also on the impact of ultrasound exposure for cell viability, proliferation rate and their function. Another author, Kin Fong Lei, in this special issue, reviewed the impedance detection of cellular response in a micro/nano environment [7]. The author 
reviewed the impact of a microfluidic system combined with the impedance measurement technique, which can provide non-invasive and label-free monitoring of cellular responses in 2D and 3D culture systems. Santra et al. [8] reviewed recent progress of micro/nanaofluidic single cell electroporation for intracellular and extracellular delivery. The electroporation technique is not only useful for cell lysis, cell to cell fusion or separation, insertion of drugs, DNA and antibodies inside single cell, but also it can control biochemical, electrical and mechanical parameters. The single cell electroporation technique can provide high transfection efficiency, higher cell viability, lower sample contamination, lower joule heating effect, and low toxicity during experiments compared to bulk measurement. As a result, single cells with their organelles can be measured more precisely by using micro/nanaofluidic devices. The authors describe in detail recent single cell and localized single cell electroporation techniques and their impact on single cell analysis.

Maorshed and coworkers [9], provided theoretical and experimental information on single cell electrical lysis in a microfluidic device and effects in a microchannel. They suggested that the generation of an electric field in a microchannel can provide sufficient energy with low current requirements. Single cell lysis in a microfluidic device was found to apply an external applied voltage approximately $700 \mathrm{~V}$ to $900 \mathrm{~V}$ within seven seconds and used less than $300 \mathrm{~mW}$ power consumption. For this single cell lysis, they used an $8 \mathrm{~mm}$ long microchannel with dimensions of $100 \mu \mathrm{m} \times 20 \mu \mathrm{m}$.

Feng et al. [10] proposed on-chip enucleation of bovine oocytes by using magnetically driven microrobot flow control. The microrobot can control the flow speed with fluid resistance adjustment in a microfluidic device and was specifically designed for enucleation. Their device can: (a) promote fluid flow control; (b) the volume of the oocyte can be adjusted resulting in less damage of the oocyte; (c) and to control microrobot and hydrodynamic forces, the nucleus can be removed. To use this device, they achieved minimally invasive enucleation with $2.5 \mathrm{~s}$ average enucleation time and $20 \%$ average removal volume ratio.

Probst et al. [11] suggested PDMS based submicron traps for single cell analysis of bacteria. The authors presented a hundred submicron size-based trapped barrier structure in a microfluidic device for immobilization and cultivation of individual bacteria. However, the study of prokaryotic cells, such as E. coli, encountered some challenges because of their small size and fast growth rates. They cultivated E. Coli for several hours within their microfluidic trapped structure, and it showed constant division times with rod-shaped morphology, indicating excellent cell growth with high cell viability.

Banaeiyan et al. [12] presents a specific microfluidic device as a cell comb, which is capable of high throughput single cell experiments. The microfluidic device can trap at least six cells in each $\mathrm{V}$ shape structure by using hydrodynamic forces giving a cellular response, such as protein migration followed by bright field and fluorescence imaging. They monitored arsenite (As (III)) uptaken in Saccharomyces cerevisiae cells with different flow rates (low $=25 \mathrm{~nL} \mathrm{~min}{ }^{-1}$, moderate $=50 \mathrm{~nL} \mathrm{~min}{ }^{-1}$, and high $=100 \mathrm{~nL} \min ^{-1}$ ). The device might be applicable for cell signaling pathways and to their modes of function and regulation.

Blomqvist et al. [13] studied single yeast cells with highly effective $\operatorname{Hog} 1$ inhibitor and the use of osmotic stress. They have used four channel microfluidic systems to enable multiple signal inputs (Hog 1 and sorbitol) to a yeast signal transduction pathway for studying single cell response. To activate the Hog 1 signaling pathways for the presence or absence of the cellular response, 
was monitored by the imaging of the nuclear translocation of the cytosolic MAPK, Hog1 on a single-cell level.

Hall et al. [14] described single cyanobacterium lysis for whole genome amplification. They present a lysis protocol, which can extract genomic information from single cyanobacterium of Synechocystis sp.PCC 6803, which have multilayer cell wall structures usually preventing the use of a conventional lysis mechanism. The high-fidelity genome sequencing of single cells of Synechocystis can be achieved by performing microfluidic MDA (Multiple Displacement Amplification) reactions with selected genes (15 loci nearly equally spaced throughout the main chromosome).

In conclusion, this special issue of Micromachines, "Micro/Nanofluidic Devices for Single Cell Analysis" not only emphasizes the new microfluidic devices for SCA, but also reviews recent advancements of SCA with different techniques such as electroporation, ultrasound, and impedance measurement. In the last couple of years, SCA has not only been at the forefront of biological cell studies and therapeutic research, but also it has been in close collaboration with human health. Recently microfabricated devices called "Laboratory on a chip" (LOC) perform a tremendous role in single cell analysis. Micro/nanofluidic devices are not only useful for cell manipulation, cell lysis, and cell separation, but also can easily control biochemical, electrical, mechanical parameters for single cell analysis. By miniaturizing the device such as in micro total analysis ( $\mu$ TAS) systems, analysis of single cell organelles with precise biochemical control can be achieved inside the single cell. SCA for system biology, genomics, transcriptomics, proteomics, metabolomics and fluxomics is not only a broad research area, but also a challenging task for application in biology, medicine, pathology and clinical trials, etc. With the continuous progress of single cell analysis, development of biomedical technologies which are essential to our daily life could be extended to offer many possibilities in the future.

\section{Author Contributions}

Tuhin Subhra Santra wrote this editorial and Fan Gang Tseng provided the concept to write an editorial and finally corrected it.

\section{Conflicts of Interest}

The authors declare no conflict of interest.

\section{References}

1. Fritzsch, F.S.O.; Dusny, C.; Frick, O.; Schmid, A. Single-cell analysis in biotechnology, systems biology, and biocatalysis. Annu. Rev. Chem. Biomol. Eng. 2012, 3, 129-155.

2. Andersson, H.; van den Berg, A. Microtechnologies and nanotechnologies for single cell analysis. Curr. Opin. Biotechnol. 2004, 15, 44-49.

3. Chiao, T-C.; Ros, A. Microfluidic single-cell analysis of intracellular compounds. J. R. Soc. Interface 2008, 5, S139-S150.

4. Lu, X.; Huang, W-H.; Wang, Z-L, Cheng, J-K. Recent developments in single cell analysis. Anal. Chim. Acta 2004, 510, 127-138. 
5. Wu, R-G.; Yang, C-S.; Cheing, C-C.; Tseng, F-G. Nanocapillary electrophoretic electrochemical chip: towards analysis of biochemicals released by single cell. J. R. Soc. Interface Focus 2011, 1, 744-753.

6. Wiklund, M.; Christakou, A.E.; Ohlin, M.; Iranmanesh, I.; Frist, T.; Vanherberghen, B.; Onfelt, B. Ultrasound-induced cell-cell interaction studies in a multi-well microplate. Micromachines 2014, 5, 27-49.

7. Lei, K.F. Review on impedance detection of cellular responses in micro/nano environment. Micromachines 2014, 5, 1-12.

8. Santra, T.S.; Tseng, F-G. Recent trends on micro/nanofluidic single cell electroporation. Micromachines 2013, 4, 333-356.

9. Morshed, B.I.; Shams, M.; Mussivand, T. Analysis of electric fields inside microchannels and single cell electrical lysis with a microfluidic device. Micromachines 2013, 4, 243-256.

10. Feng, L.; Hagiwara, M.; Ichikawa, A.; Arai, F. On-chip enucleation of bovine oocytes using microrobot-assisted flow-speed control. Micromachines 2013, 4, 227-285.

11. Probst, C.; Grunberger, A.; Weichert, W.; Kohlheyer, D. Polydimethylsiloxane (PDMS) sub-micron traps for single-cell analysis of bacteria. Micromachines 2013, 4, 357-369.

12. Banaeiyan, A.B.; Ahmadpour, D.; Adiels, C.B.; Goksor, M. Hydrodynamic cell trapping for high throughput single-cell applications. Micromachines 2013, 4, 414-430.

13. Blomqvist, C.H.; Diner, P.; Grotli, M.; Goksor, M.; Adiels, C.B. A single-cell study of a highly effective Hog1 inhibitor for in situ yeast cell manipulation. Micromachines 2014, 5, 81-96.

14. Hall, E.W.; Kim, S.; Appadoo, V.; Zare, R.N. Lysis of a single cyanobacterium for whole genome amplification. Micromachines 2013, 4, 321-332.

(C) 2014 by the authors; licensee MDPI, Basel, Switzerland. This article is an open access article distributed under the terms and conditions of the Creative Commons Attribution license (http://creativecommons.org/licenses/by/3.0/). 